any HPN cohort is vital, given the associated risk of HPN related complications. Moreover, with developments in surgical lengthening and potential for emerging pharmacological interventions, appropriate patient selection is key. However, there may be regional and national differences between different SBS-IF patient populations; this study therefore aimed to develop a greater contemporary understanding of the SBS-IF subset managed within a large U. K. HPN cohort.

Method We performed a retrospective observational study from a prospectively maintained database, evaluating patients with type 3 IF managed in a national U. K. centre. Patients' intestinal anatomical details were reviewed and PN requirements evaluated according to the novel ESPEN classification for type 3 IF. Each individual SBS case was evaluated to assess eligibility for GLP-2 analogue therapy according to recently published inclusion criteria

Results A total of 273 patients were included in the HPN database as of October 2017. One hundred and fifty two patients (55.7\%; mean age of 56.9 years) were identified as having IF as a result of SBS (SBS-IF), with the presence of a jejunostomy (SBS-J; 41.8\%) as the most frequent pathophysiological mechanism. Only $7.3 \%$ of patients with SBS-IF had colon in continuity. Crohn's disease was the most common underlying aetiology leading to SBS-IF. The mean duration of HPN was 60.8 months (range: 4-415.8). Univariate analysis for the whole HPN cohort demonstrated SBS-IF and a longer duration of HPN to be associated with higher PN energy requirements, $\mathrm{p} \leq 0.0001$. Seventy three $(48.0 \%)$ patients with SBS-IF were deemed suitable for treatment with a GLP-2 analogue, with co-morbidity being the most frequent cause of non-suitability.

Conclusion This is the largest UK HPN cohort individually reported using ESPEN pathophysiological and clinical severity classification. The vast majority of patients with SBS-IF have a jejunostomy and, as compared to other international cohorts, relatively few have colon-in-continuity. The study further demonstrates that existing co-morbidity is a principal contra-indication to therapy with GLP-2 analogue therapy in a majority of patients with SBS-IF; these data will be useful for funding bodies to consider when planning reimbursement costs for novel therapies within limited national healthcare budgets.

\section{ADWE-09 LOW FODMAP DIET IMPROVES FUNCTIONAL-LIKE GASTROINTESTINAL SYMPTOMS BUT REDUCES BIFIDOBACTERIA IN QUIESCENT INFLAMMATORY BOWEL DISEASE}

\begin{abstract}
${ }^{1}$ Selina Cox* ${ }^{2}$ Andrew Stagg, ${ }^{3}$ Sebastién Fromentin, ${ }^{1,3}$ Dusko Ehrlich, ${ }^{2}$ Neil McCarthy, ${ }^{3}$ Nathalie Galleron, ${ }^{3}$ Florence Levenez, ${ }^{1,4}$ Miranda Lomer, ${ }^{5}$ James Lindsay, ${ }^{4}$ Peter Irving, ${ }^{1}$ Kevin Whelan. 'King's College London, London, UK; ${ }^{2}$ Queen Mary University of London, London, UK; ${ }^{3}$ Institut National de la Recherche Agronomique, Paris, France; ${ }^{4}$ Guy's and St Thomas' NHS Foundation Trust, London, UKi ${ }^{5}$ Barts Health NHS Trust, London, UK
\end{abstract}

\subsection{6/gutjnl-2018-BSGAbstracts.328}

Introduction Many patients with quiescent inflammatory bowel disease (IBD) also experience functional-like GI symptoms. The low FODMAP diet improves GI symptoms in quiescent IBD in uncontrolled trials but there are no placebo-controlled trials to confirm this. We performed a randomised, placebocontrolled trial to assess the effects of a low FODMAP diet on GI symptoms, microbiota, inflammatory markers and circulating gut-tropic $(\alpha 4 \beta 7+)$ T-cells in patients with quiescent IBD.
Methods Patients with Crohn's disease (CD) or ulcerative colitis (UC) were included. Quiescent IBD was defined as: 1) Physician Global Assessment, 2) faecal calprotectin [FC] $<250 \mu \mathrm{g} / \mathrm{g}$ and 3) CRP $<10 \mathrm{mg} / \mathrm{L}$. Suitable patients fulfilled the Rome III criteria for IBS, functional bloating or functional diarrhoea, and were naïve to the low FODMAP diet. Participants were randomised to low FODMAP or placebo (sham) dietary advice for 4 weeks. At baseline and end of trial, GI symptoms and stool output were measured using validated questionnaires. Faecal microbiota were characterised using metagenomic sequencing and $\alpha 4 \beta 7+\mathrm{T}$ cell populations quantified using flow cytometry. End of trial data were compared intention to treat between the diets using analysis of covariance adjusting for baseline values.

Results Fifty two patients were randomised (27 low FODMAP diet, 25 sham diet). At the end of trial, more patients reported adequate relief of GI symptoms following the low FODMAP diet $(14 / 27,52 \%)$ than the sham diet $(4 / 25,16 \%)$ $(p=0.007)$. Total IBS Severity Scoring System score decreased by 67 points (SD 78) during the low FODMAP diet and 34 points (SD 50) during the sham diet $(p=0.075)$. Daily stool frequency was lower following low FODMAP diet (1.7 SD $0.5)$ than sham diet $(2.1 \mathrm{SD} 0.5)(\mathrm{p}=0.012)$. Bacterial gene richness was not different between the groups at end of trial $(\mathrm{p}=0.620)$. Relative abundance of Bifidobacterium longum $\left(1.24-^{7}\right.$ vs $\left.6.95-^{7}, \mathrm{p}=0.003\right)$ and $B$. adolescentis $\left(1.99-^{7}\right.$ vs $\left.2.55-^{6}, \quad p=0.015\right)$ was lower following low FODMAP diet compared to sham diet. Between baseline and end of trial, Faecalibacterium prausnitzii SL3/3 M21/2 (2.30-6 vs $1.52-^{6}$, $\mathrm{p}=0.029)$ and $F$. prausnitzii KLE1255 (4.49- ${ }^{6}$ vs. $2.68{ }^{6}$ ' $p=0.006$ ) declined in the low FODMAP diet group. There was no difference in proportions of $\alpha 4 \beta 7+\mathrm{T}$ cells between groups at end of trial.

Conclusions The low FODMAP diet improved functional-like GI symptoms in patients with quiescent IBD but reduces immunoregulatory species of the intestinal microbiota, though does not impact on inflammatory markers or $\alpha 4 \beta 7+$ blood Tcell numbers.

\section{PWE-095 WHAT IS THE ROLE OF CAPSULE ENDOSCOPY IN EVALUATING PATIENTS WITH REFRACTORY COELIAC DISEASE?}

Stefania Chetcuti Zammit*, David S Sanders, Reena Sidhu. Sheffield Teaching Hospitals, Sheffield, UK

\subsection{6/gutjnl-2018-BSGAbstracts.329}

Introduction Small bowel capsule endoscopy (SBCE) is used in refractory coeliac disease (RCD) to assess the extent of disease and ensure there are no complications (lymphoma or ulcerative jejunitis). However there are no published reports on SBCE in RCD following immunosuppressive therapy.

Methods Patients with histologically confirmed refractory coeliac disease (RCD) who underwent a SBCE at baseline and after treatment were enrolled in this study. These were compared to a group of control CD patients with no underlying RCD.

Results 19 patients (median 53 years) with RCD (12 patients; $63.2 \%$ - type 1) were compared to 28 patients with control CD (median 48 years). There was no statistically significant difference in duration of disease, gender, age at SBCE and serology between the 2 groups. 
Patients with RCD were more likely to have worse histology (Marsh 3a-c) than SBCE. control CD who had a higher percentage of normal histology at the time of SBCE. $(p=0.002)$ Those with RCD had a longer abnormal small bowel (SB) mucosa (185 SD \pm 167.6 vs 29.5 SD $\pm 73 \mathrm{~min}$ $\mathrm{p}=0.0001)$ and longer percentage of abnormal SB (53.9 $\mathrm{SD} \pm 38.0$ vs $6.9 \mathrm{SD} \pm 15.2 \mathrm{~min} \mathrm{p}=0.0001$ ) when compared to those with control CD.

A repeat SBCE was carried out after a mean of 9.63 $\mathrm{SD} \pm 6.6$ months in patients with RCD following treatment. There was no statistical significant difference in histology and serology at the time of the first and second SBCE. Patients received the following treatment: $36.8 \%$ steroids, $26.3 \%$ immunosuppressants, $36.1 \%$ combination of mycophenolate azathioprine and steroids. However, there was an improvement in the length of abnormal SB mucosa (185 vs 116 min; $\mathrm{p}=0.035)$ and the percentage of abnormal SB (50.8 vs $32.9 \%$; $\mathrm{p}=0.027) .7$ patients $(36.8 \%)$ had diffuse mucosal involvement on the first SBCE but only 4 (21.1\%) had diffuse disease on repeat SBCE. $(p=0.007)$ There was no statistical correlation between coeliac serology and small bowel passage time, length of mucosal abnormality and percentage of affected SB at first and second SBCE. The same findings were also true for histology.

Conclusions More severe SB involvement on SBCE can be found in patients with RCD. This is the first study that shows an improvement in SB abnormality on SBCE following treatment of RCD patients. Histology is useful in distinguishing RCD from non-RCD but not for assessing improvement in patients with RCD following treatment. SBCE might potentially be regarded as a less invasive, more accurate way of following up these patients.

\section{PWE-096 WHAT IS THE COST OF DELAYED DIAGNOSIS OF BILE ACID MALABSORPTION?}

Darren Fernandes*, Dennis Poon, Laura White, Jervoise Andreyev. Lincoln County Hospital, Lincoln, UK

\subsection{6/gutjnl-2018-BSGAbstracts.330}

Introduction Bile acid malabsorption (BAM) is accurately diagnosed using a ${ }^{75}$ selenium taurocholic acid (SeHCAT) scan which also defines treatments patients require. BAM causes chronic, often debilitating symptoms including loose stool, faecal incontinence and abdominal pain. Primary BAM affects $1 \%$ of Britons yet is frequently misdiagnosed as IBS. A further $1 \%$ have BAM secondary to other conditions. The 2012 NICE DG7 review of SeHCAT included a cost-effectiveness evaluation based on assumptions without supporting evidence. Our aim was to evaluate the cost of delayed diagnosis of BAM.

Methods The notes of all patients undergoing SeHCAT scanning in our Trust over a one-year period were reviewed retrospectively. The number of abnormal scans and patient response to treatment were recorded. Costs of additional clinics/tests/procedures performed before the diagnosis of BAM were calculated using NICE costing templates.

Results $1.5 \%$ of 3860 new patients seen in our gastroenterology clinics between June 2016-May 2017, 19 men and 37 women, median age 58 (range 19-83) were referred for SeHCAT scanning. Of these, 64\% were abnormal: 13 demonstrated severe $(<5 \% 7$ day SeHCAT retention), 13 moderate
(5\%-10\%), 5 mild (10\%-15\%) and 5 borderline (15\%-20\%) BAM. Underlying causes for BAM included cholecystectomy $(n=13)$, chronic unexplained symptoms $(n=12)$, inflammatory bowel disease $(n=4)$, irritable bowel syndrome $(n=4)$, right hemicolectomy for bowel cancer $(n=1)$, diabetes mellitus $(n=1)$ and multiple possible causes $(n=1)$. If SeHCAT scanning was ordered at first consultation $(n=11)$, patients reported 24 months (median) of symptoms (range 6-360) and the mean diagnostic package of care cost was $£ 910.75$. If the SeHCAT scan booked 2nd line or later $(n=25)$, patients reported symptoms for 30 months (median, range 0.5-360) and mean diagnostic package of care cost was $£ 1,481.73$. However, in these patients 9 additional abnormalities were found: vitamin D deficiency $(n=3)$, diverticulosis $(n=2)$, folate deficiency $(n=1)$, oesophageal dysmotility $(n=1)$, renal cell carcinoma requiring nephrectomy $(n=1)$ and Helicobacter Pylori gastritis $(n=1)$. Following diagnosis, treatment led to reported symptom improvement $(n=24)$, no change/deterioration $(n=3)$, not reported $(n=9)$.

Conclusions In our Trust, SeHCAT scanning is enormously underused. Late diagnosis of BAM is associated with markedly increased costs, unnecessary demands for other services and treatment delay for patients. National data on SeHCAT usage suggest that our findings will apply to most other Trusts. More emphasis to ensure early diagnosis of BAM, a frequent, unpleasant and treatable condition would bring the unusual but highly desirable result of significant health benefits while substantially reducing healthcare costs.

\section{PWE-097 OUTCOMES OF ROUTINE TUBERCULOSIS TESTING WHEN ILEITIS IS DIAGNOSED IN A HIGH INCIDENT TB AREA}

1,2Misha Kabir*, 'Harry Coleman, ${ }^{1}$ Nivenka Jayasekera, ${ }^{1}$ Noor Jawad. 'Newham Hospital, Barts Health NHS Trust, London, UK; ${ }^{2}$ St Mark's Hospital, London, UK

\subsection{6/gutjnl-2018-BSGAbstracts.331}

Introduction In comparison to the UK's low average incidence of Tuberculosis (TB) at 9.9 per 100000 population (2), the London Borough of Newham stands out as having the highest UK 3 year average annual incidence at 69.5 per 100000 population (1), which is on par with some endemic countries in Asia and Africa (2). This is due to its predominance of non-UK born residents and socio-economic deprivation. It can often be difficult to differentiate between Crohn's disease (CD) and TB ileitis and missed cases of intestinal TB treated with immunosuppression could potentially have devastating consequences. We proposed a service change in Newham University Hospital routinely testing for $\mathrm{TB}$ in any case of ileitis found at colonoscopy, and evaluate its impact on TB detection.

Method Before the study period, all endoscopists performing colonoscopies at Newham University Hospital were instructed to routinely send a TI biopsy sample for Ziel-Nielson staining for Acid-fast Bacilli (AFB) and in saline for TB culture, if they found macroscopic inflammatory changes suggestive of terminal ileitis (such as ulceration or erythema). Data was collected from patient records for all ileocolonoscopies performed between 3rd October 2016 and 5th May 2017.

Results The TI was visualised in $46.2 \%$ of the 649 colonoscopies recorded. 25 cases of macroscopic terminal ileitis were reported $(8.3 \%$ of total ileocolonoscopies). $40 \%$ of the 